\title{
CONTACT AND CHORD LENGTH DISTRIBUTION FUNCTIONS OF THE POISSON-VORONOI TESSELLATION IN HIGH DIMENSIONS
}

\author{
L. MUCHE, ${ }^{*}$ Fraunhofer Institute for Integrated Circuits
}

\begin{abstract}
In this paper we present formulae for contact distributions of a Voronoi tessellation generated by a homogeneous Poisson point process in the $d$-dimensional Euclidean space. Expressions are given for the probability density functions and moments of the linear and spherical contact distributions. They are double and simple integral formulae, which are tractable for numerical evaluation and for large $d$. The special cases $d=2$ and $d=3$ are investigated in detail, while, for $d=3$, the moments of the spherical contact distribution function are expressed by standard functions. Also, the closely related chord length distribution functions are considered.
\end{abstract}

Keywords: Probability density function; moment; linear and spherical contact distribution functions; chord length

2000 Mathematics Subject Classification: Primary 60D05

Secondary 52A22

\section{Introduction}

The Voronoi tessellation is a popular stochastic geometrical model applied in many fields of science and engineering; see, e.g. [9]. A commonly used special case is that of complete randomness in which the generating point pattern is a homogeneous Poisson point process. This so-called Poisson-Voronoi tessellation has been studied by many researchers, both analytically and by means of simulation.

Contact distributions are important random set summary characteristics. They describe the size of pores or porous space; see, e.g. [4] and [5]. In the context of a tessellation they can be used to characterize the size and the shape of cells; the random set of interest is then the union of all cell boundaries.

A first analytical approach to the linear contact distribution function of the planar and spatial Poisson-Voronoi tessellations is given by Gilbert [2], while Serra [11, p. 527] presented a formula for the spherical contact distribution function for the spatial Poisson-Voronoi tessellation. Muche and Stoyan [8] provided a unified method to calculate linear and spherical contact distribution functions of the planar and spatial Poisson-Voronoi tessellations. This paper also gave an analytical expression for the chord length distribution function, using the close relationship to the linear contact distribution function. Furthermore, the problem of calculation of chord length distribution functions in higher dimensions $d$ was mentioned there.

Other analytical approaches to investigate the chord length distribution function in higher dimensions are given in Schlather [10] and Muche [7]. In these papers formulae are presented

Received 8 April 2009; revision received 6 October 2009.

* Postal address: Fraunhofer Institute for Integrated Circuits, EAS Dresden, Zeunerstraße 38, D-01069 Dresden, Germany. Email address: lutz.muche@eas.iis.fraunhofer.de 
for the probability density function in double integral form, which are tractable for numerical calculations.

Alishahi and Sharifitabar [1] studied the linear contact and chord length distribution functions of the Poisson-Voronoi tessellation for large $d$. The limiting functions of both characteristics $H_{l}^{\infty}(r)$ and $L^{\infty}(r)$ (for $d \rightarrow \infty$ ) were given in terms of standard functions. The value of the density function of $L^{\infty}(r)$ for $r=0$ was given explicitly. Plots of the density function for various $d$ were obtained by simulation.

In further papers, contact and chord length distribution functions are studied for some modificated Voronoi tessellations; cf. [3] and [6].

In the present paper we investigate the contact and chord length distribution functions of the Poisson-Voronoi tessellation of arbitrary dimension $d$. These calculations are mainly based on the methods provided in Muche and Stoyan [8] and Muche [7]. The probability density functions $h_{l_{d}}(r)$ and $f_{l_{d}}(r)$ (the notation is explained in Section 2) of both characteristics and their moments are given in double integral form, tractable for an efficient numerical evaluation and for large dimension $d$. Expressions are given for $h_{l_{d}}(0)$ explicitly and for $f_{l_{d}}(0)$ in simple integral form for arbitrary $d$. A new approach is used to determine the spherical contact distribution function in higher dimensions. Probability density functions and moments are given in simple integral form, tractable for numerical calculations and for large $d$. For the special case $d=3$, the moments of the spherical contact distribution functions are given by standard functions. A simple relationship between linear and spherical contact distribution functions is presented.

The paper is organized as follows. In Section 2 we introduce the notation, give the definitions of the contact and chord length distribution functions, and sketch a means to calculate them. The main results are given in Sections 3 and 4. In Section 5 the spherical contact distribution functions are investigated in particular for the special cases $d=2$ and $d=3$. Section 6 gives the proofs of all the results.

\section{Fundamentals}

Let $\Phi$ denote a homogeneous Poisson point process of intensity $\lambda$ in the $d$-dimensional Euclidean space $\mathbb{R}^{d}, 2 \leq d<\infty$. Let $z_{0}, z_{1}, z_{2}, \ldots$ be its points, also called centres, neighbours, seeds, or atoms. Let $\mathscr{H}_{n}$ be any section subspace of dimension $n(1 \leq n<d)$ through $\mathbb{R}^{d}$. Let $u, x$, and $y$ denote elements of $\mathbb{R}^{d}$, and let $\|x-y\|$ denote the Euclidean distance (norm) between two of them. Let $\mathcal{V}$ be the Voronoi tessellation generated by $\Phi$. The cells of $\mathcal{V}$ are $d$-dimensional bounded open convex polytopes. Let $C_{0}$ denote that cell containing the origin $o$, and let $z_{0}$ denote the generating point of $C_{0}$. Let us denote by $\Xi$ the set of all cell boundaries (vertices, edges, $\ldots,(d-1)$-dimensional cell faces), or, equivalently, the set of all elements not inside a cell. Since $\Phi$ is stationary and isotropic, so is $\Xi$. Let $s(x, r)$ be the line segment of given direction (positive $x_{1}$-axis) of length $r$ starting in $x$, let $b(x, r)$ be the ball of radius $r$ centred in $x$, and let $\partial b(x, r)$ be its boundary; $r s(x, 1)$ and $r b(x, 1)$ are the same as $s(x, r)$ and $b(x, r)$, respectively. Furthermore, let $f^{d-1}=\partial b(o, 1)$ be the $(d-1)$-dimensional unit sphere, and let $\sigma$ and $w$ be elements of $\delta^{d-1}$. Let $\omega_{d}$ denote the volume of the $d$-dimensional unit ball $b(o, 1)$,

$$
\omega_{d}=\frac{\pi^{d / 2}}{\Gamma(d / 2+1)},
$$

where $\Gamma(t)$ is Euler's $\Gamma$-function. 
The contact distribution function $H_{B}(r)$ is defined as

$$
H_{B}(r)=1-\frac{\mathrm{P}(\Xi \cap r B=\varnothing)}{1-\mathrm{P}(o \in \Xi)}, \quad r \geq 0,
$$

where $B$ is a specified test set or structuring element, a subset of $\mathbb{R}^{d}$ with $o \in B$. In other words, $H_{B}(r)$ is the probability that $r B$ intersects $\Xi$, conditional on $\Xi$ not containing $o$. For a definition, further representations, and basic properties, see [12, p. 71ff.].

Two important cases are

- the linear contact distribution function $H_{l}(r)$, where $B=s(o, 1)$ is the unit segment,

- the spherical contact distribution function $H_{S}(r)$, where $B=b(o, 1)$ is the unit ball.

The chord length distribution function $F_{l}(r)$ of a random tessellation is the distribution function of the interval lengths formed by the intersections of $\Xi$ with a fixed line; cf. [12, p. 82]. Furthermore, there is the close relationship between $H_{l}(r)$ and $F_{l}(r)$ :

$$
H_{l}(r)=\frac{1}{\mathrm{E} L} \int_{0}^{r}\left(1-F_{l}(t)\right) \mathrm{d} t
$$

(cf. [12, p. 208]), where $\mathrm{E} L$ is the mean chord length.

Following the results given in Muche and Stoyan [8], the contact distribution function can be written in general form as

$$
H_{B}(r)=1-\int_{0}^{\infty} \int_{\delta^{d-1}} \exp \left\{-\lambda v_{d}\left(U_{r, \rho, \sigma} \backslash b(o, \rho)\right)\right\} f_{P}(\rho) f_{\Sigma}(\sigma) \mathrm{d} \sigma \mathrm{d} \rho,
$$

with polar coordinates $(\rho, \sigma), 0 \leq \rho<\infty$ and $\sigma \in 8^{d-1}, d$-volume $v_{d}(\cdot)$, and

$$
U_{r, \rho, \sigma}=\bigcup_{x \in r B} b(x,\|(\rho, \sigma)-x\|) .
$$

The exponential expression in (2) describes the probability that the test $\operatorname{set} r B$ is completely included in the origin cell $C_{0}$ under the condition that its generating point is $z_{0}=(\rho, \sigma)$. The distance between $o$ and $z_{0}$ is characterized by the well-known nearest-neighbour distribution function. Its density function is

$$
f_{P}(\rho)=\lambda d \omega_{d} \rho^{d-1} \exp \left\{-\lambda \omega_{d} \rho^{d}\right\}, \quad 0 \leq \rho<\infty .
$$

Let $\Sigma$ denote the ray emanating from $o$ and passing through $z_{0}$. Based on the isotropy of $\mathcal{V}, \Sigma$ is uniformly distributed on $8^{d-1}$, i.e. its density function is

$$
f_{\Sigma}(\sigma)=\frac{1}{d \omega_{d}}
$$

For further investigations, the $d$-volume of two overlapping (or truncated) balls is needed. Following Muche [7, Equations (8), (9), and (10)], the $d$-volume of two overlapping balls with midpoint distance $r$ and radii $r \sin \alpha / \sin (\alpha+\beta)$ and $r \sin \beta / \sin (\alpha+\beta)$ (as $\rho$ and $\sigma$, and $\alpha$ and $\beta$, are random variables, called intersection longitudes) is given by

$$
v_{d}(r, \alpha, \beta)=\omega_{d} r^{d}\left(\frac{\sin ^{d} \beta}{\sin ^{d}(\alpha+\beta)} \sum_{i=0}^{\lfloor d / 2\rfloor} a_{i}(\alpha)+\frac{\sin ^{d} \alpha}{\sin ^{d}(\alpha+\beta)} \sum_{i=0}^{\lfloor d / 2\rfloor} a_{i}(\beta)\right),
$$


where $\lfloor\theta\rfloor$ denotes the greatest integer smaller than $\theta$ and

$$
\begin{aligned}
& a_{0}(\psi)=\left\{\begin{array}{l}
1-\frac{\psi}{\pi}, \quad d \text { even, } \\
\cos ^{2} \frac{1}{2} \psi, \quad d \text { odd, }
\end{array}\right. \\
& a_{i}(\psi)=\frac{\cos \psi}{2 \sqrt{\pi}}\left\{\begin{array}{l}
\frac{\Gamma(i)}{\Gamma(i+1 / 2)} \sin ^{2 i-1} \psi, \quad d \text { even, } \quad i=1, \ldots,\left\lfloor\frac{1}{2} d\right\rfloor . \\
\frac{\Gamma(i+1 / 2)}{\Gamma(i+1)} \sin ^{2 i} \psi, \quad d \text { odd, }
\end{array}\right.
\end{aligned}
$$

Alternatively, with midpoint distance $r$ and radii $\rho$ and $\sqrt{r^{2}+\rho^{2}-2 r \rho \cos \alpha}$, the $d$-volume is

$$
\begin{aligned}
v_{d}(r, \alpha, \beta(r, \rho, \alpha))=\omega_{d}( & \rho^{d} \sum_{i=0}^{\lfloor d / 2\rfloor} a_{i}(\alpha) \\
& \left.+\left(r^{2}+\rho^{2}-2 r \rho \cos \alpha\right)^{d / 2} \sum_{i=0}^{\lfloor d / 2\rfloor} a_{i}\left(\operatorname{arccot} \frac{r-\rho \cos \alpha}{\rho \sin \alpha}\right)\right) .
\end{aligned}
$$

Both expressions (5) and (7) are related by the formulae

$$
\beta=\operatorname{arccot} \frac{r-\rho \cos \alpha}{\rho \sin \alpha}
$$

and

$$
\rho=\frac{r \sin \beta}{\sin (\alpha+\beta)},
$$

and the corresponding partial derivatives

$$
\begin{gathered}
\frac{\partial \beta}{\partial \rho}=\frac{r \sin \alpha}{r^{2}+\rho^{2}-2 r \rho \cos \alpha}, \\
\frac{\partial \rho}{\partial \beta}=\frac{r \sin \alpha}{\sin ^{2}(\alpha+\beta)} .
\end{gathered}
$$

The first derivative of $v(r, \alpha, \beta)$ with respect to $r$ transformed according to (9), elaborated in detail in Muche [7, p. 293], is

$$
v_{d}^{\prime}(r, \alpha, \beta)=d \omega_{d}\left(\frac{r \sin \alpha}{\sin (\alpha+\beta)}\right)^{d-1} \sum_{i=0}^{\lfloor(d-1) / 2\rfloor} b_{i}(\beta),
$$

with

$$
\begin{aligned}
& b_{0}(\psi)= \begin{cases}\frac{(\pi-\psi) \cos \psi+\sin \psi}{\pi}, & d \text { even, } \\
\cos ^{2} \frac{1}{2} \psi, & d \text { odd, }\end{cases} \\
& b_{i}(\psi)=-\frac{1}{4 \sqrt{\pi}} \begin{cases}\frac{\Gamma(i)}{\Gamma(i+3 / 2)} \sin ^{2 i+1} \psi, & d \text { even, } \\
\frac{\Gamma(i-1 / 2)}{\Gamma(i+1)} \sin ^{2 i} \psi, & d \text { odd },\end{cases}
\end{aligned}
$$


In the same way, the second derivative with respect to $r$ is given by

$$
v_{d}^{\prime \prime}(r, \alpha, \beta)=d \omega_{d}\left(\frac{r \sin \alpha}{\sin (\alpha+\beta)}\right)^{d-2\lfloor(d-3) / 2\rfloor} \sum_{i=0} c_{i}(\beta)
$$

with

$$
\begin{gathered}
c_{0}(\psi)= \begin{cases}\frac{d-1}{\pi}\left(\left(1-\frac{d-2}{d-1} \sin ^{2} \psi\right)(\pi-\psi)+\sin \psi \cos \psi\right), & d \text { even, } \\
\frac{1}{2}(1+\cos \psi)(1+(d-2) \cos \psi), & d \text { odd },\end{cases} \\
c_{i}(\psi)=-\frac{1}{4 \sqrt{\pi}} \begin{cases}\frac{\Gamma(i)}{\Gamma(i+3 / 2)}(d-2 i-2) \sin ^{2 i+1} \psi \cos \psi, & d \text { even } \\
\frac{\Gamma(i-1 / 2)}{\Gamma(i+1)}(d-2 i-1) \sin ^{2 i} \psi \cos \psi, & d \text { odd }\end{cases}
\end{gathered}
$$

for $i=1, \ldots,\left\lfloor\frac{1}{2}(d-3)\right\rfloor$. Furthermore, knowledge of the density function of one of the angles $\alpha$ and $\beta$ occurring in (5) and (7) is necessary. Consider an element $\sigma \in \varsigma^{d-1}$ and an $n$-dimensional section subspace $\mathscr{H}_{n}(1 \leq n<d), o \in \mathscr{H}_{n}$. Let $\sigma^{\prime}$ be the projection of $\sigma$ on $\mathscr{H}_{n}$, and let $A_{d, n}$ be the angle $\angle\left(\sigma o \sigma^{\prime}\right)$. If $\sigma$ is uniformly distributed on $\delta^{d-1}$, the probability density function of $A_{d, n}$ is

$$
f_{A_{d, n}}(\alpha)=\frac{2 \Gamma(d / 2)}{\Gamma(n / 2) \Gamma((d-n) / 2)} \sin ^{d-n-1} \alpha \cos ^{n-1} \alpha, \quad 0 \leq \alpha<\frac{\pi}{2},
$$

as is shown in Section 6.

The assumptions used to calculate the contact and chord length distribution functions in arbitrary dimension $d$ are now complete. Henceforth, let $H_{l_{d}}(r)$ and $F_{l_{d}}(r)$ be the linear contact distribution and the chord length distribution functions of $\mathcal{V}$ in $\mathbb{R}^{d}$, respectively. Let $H_{s_{d, n}}(r)$ be the spherical contact distribution function of an $n$-dimensional section through $\mathcal{V}$ in $\mathbb{R}^{d}$. Furthermore, let $h_{l_{d}}(r), f_{l_{d}}(r)$, and $h_{s_{d, n}}(r)$ be the corresponding density functions, and let $\mathrm{E}_{l} R_{d}^{k}, \mathrm{E} L_{d}^{k}$, and $\mathrm{E}_{s} R_{d, n}^{k}, k=0,1,2, \ldots$, be the corresponding moments.

\section{The linear contact distribution function and the chord length distribution function}

This section contains the main results of this paper. All numbered equations represent new results that are valid for all dimensions $d, 2 \leq d<\infty$. These formulae are proved in Section 6 .

The integral evaluations in this section and the next section were made using MATHEMATICA $^{\circledR}$. To evaluate the probability density curves and moments for $d \rightarrow \infty$, the obtained numerical results for large $d$ were fitted by smooth auxiliary functions of $d$, which were then extrapolated to $d \rightarrow \infty$.

The probability density function of the linear contact distribution function is given by

$$
\begin{aligned}
h_{l_{d}}(r)=\frac{2 \pi^{(d-1) / 2} \lambda^{2}}{\Gamma((d-1) / 2)} \int_{0}^{\infty} \int_{0}^{\pi} & \rho^{d-1} \sin ^{d-2} \alpha \frac{\mathrm{d}}{\mathrm{d} r}\left(v_{d}(r, \alpha, \beta(r, \rho, \alpha))\right) \\
& \times \exp \left\{-\lambda v_{d}(r, \alpha, \beta(r, \rho, \alpha))\right\} \mathrm{d} \alpha \mathrm{d} \rho,
\end{aligned}
$$


or, equivalently, in terms of the angles $\alpha$ and $\beta$,

$$
\begin{aligned}
h_{l_{d}}(r)=\frac{2^{d} \pi^{d-1} \lambda^{2}}{\Gamma(d-1)} \int_{0}^{\pi} \int_{0}^{\pi-\alpha} & r^{2 d-1} \frac{\left(\sin ^{2} \alpha \sin \beta\right)^{d-1}}{\sin ^{2 d}(\alpha+\beta)} \\
& \times \sum_{i=0}^{\lfloor(d-1) / 2\rfloor} b_{i}(\beta) \exp \left\{-\lambda \nu_{d}(r, \alpha, \beta)\right\} \mathrm{d} \beta \mathrm{d} \alpha .
\end{aligned}
$$

Its value for $r=0$ is

$$
h_{l_{d}}(0)=\frac{2 \Gamma(d) \Gamma(2-1 / d)\{\Gamma((d+2) / 2)\}^{2-1 / d} \lambda^{1 / d}}{d \Gamma^{2}((d+1) / 2) \Gamma(d-1 / 2)}, \quad 2 \leq d<\infty
$$

with the limit

$$
\lim _{d \rightarrow \infty} h_{l_{d}}(0)=\sqrt{2 \mathrm{e}} \approx 2.331644
$$

See Figure 1. The moments corresponding to $H_{l_{d}}$ are

$$
\begin{aligned}
\mathrm{E}_{l} R_{d}^{k}= & \frac{2\{\Gamma((d+2) / 2)\}^{1+k / d} \Gamma(2+k / d)}{\pi^{(k+1) / 2} \Gamma((d-1) / 2) \lambda^{k / d}} \\
& \times \int_{0}^{\pi} \int_{0}^{\pi-\alpha} \frac{\left(\sin ^{2} \alpha \sin \beta\right)^{d-1} \sin ^{k}(\alpha+\beta) \sum_{i=0}^{\lfloor(d-1) / 2\rfloor} b_{i}(\beta)}{\left\{\sin ^{d} \beta \sum_{i=0}^{\lfloor d / 2\rfloor} a_{i}(\alpha)+\sin ^{d} \alpha \sum_{i=0}^{\lfloor d / 2\rfloor} a_{i}(\beta)\right\}^{2+k / d}} \mathrm{~d} \beta \mathrm{d} \alpha
\end{aligned}
$$

for $k=0,1,2, \ldots$ See Table 1 .

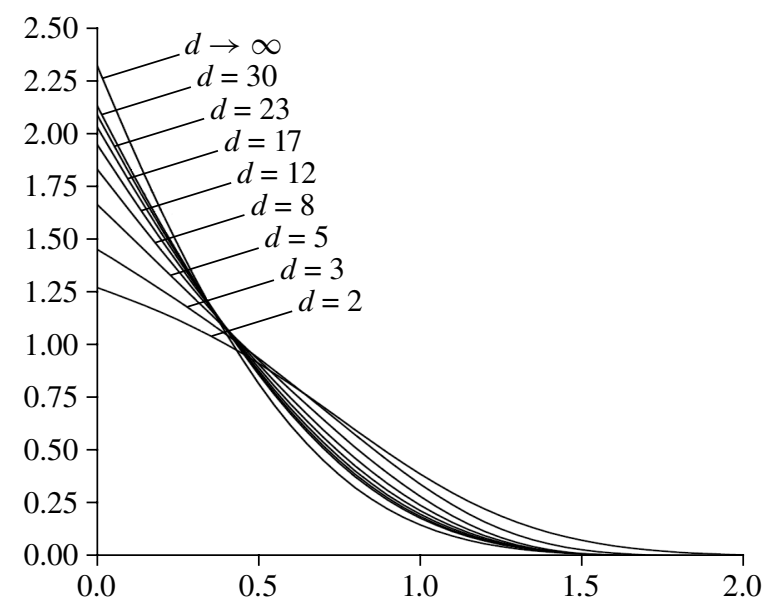

Figure 1: The probability density function of the linear contact distribution of the Poisson-Voronoi tessellation in $\mathbb{R}^{d}, d=2,3,5,8,12,17,23,30, \infty$. For all figures in this paper, the intensity of the generating Poisson point process is 1 . 
TABLE 1: Moments $\mathrm{E}_{l} R_{d}^{k}$ of the linear contact distribution function of the Poisson-Voronoi tessellation in $\mathbb{R}^{d}, d=2,3,5,8,12,17,23,30, \infty$. For all the tables in this paper, the intensity of the generating Poisson point process is 1 .

\begin{tabular}{clllll}
\hline \multirow{5}{*}{$d$} & \multicolumn{5}{c}{$k$} \\
\cline { 2 - 6 } & \multicolumn{1}{c}{1} & \multicolumn{1}{c}{2} & \multicolumn{1}{c}{3} & \multicolumn{1}{c}{4} & \multicolumn{1}{c}{5} \\
\hline 2 & 0.513166 & 0.407493 & 0.405319 & 0.468526 & 0.606279 \\
3 & 0.459153 & 0.323650 & 0.281473 & 0.279760 & 0.306145 \\
5 & 0.418703 & 0.273142 & 0.220661 & 0.203466 & 0.205787 \\
8 & 0.394841 & 0.247698 & 0.194712 & 0.176027 & 0.175422 \\
12 & 0.380181 & 0.233204 & 0.181214 & 0.163290 & 0.163265 \\
17 & 0.370603 & 0.224011 & 0.172956 & 0.155899 & 0.156814 \\
23 & 0.364012 & 0.217743 & 0.167374 & 0.150967 & 0.152633 \\
30 & 0.359283 & 0.213249 & 0.163360 & 0.147400 & 0.149588 \\
$\rightarrow \infty$ & 0.33816 & 0.19262 & 0.14400 & 0.12876 & 0.13136 \\
\hline
\end{tabular}

The probability density function of the chord length distribution function is given by

$$
\begin{aligned}
f_{l_{d}}(r)= & \frac{d^{2}(d-1) \pi^{d-1 / 2} \Gamma(d-1 / 2) \Gamma((d+1) / 2) \lambda^{2-1 / d}}{2 \Gamma(d) \Gamma(2-1 / d)\{\Gamma((d+2) / 2)\}^{3-1 / d}} \\
& \times \int_{0}^{\pi} \int_{0}^{\pi-\alpha} r^{2 d-2} \frac{\sin ^{2 d-3} \alpha \sin ^{d-1} \beta}{\sin ^{2 d-1}(\alpha+\beta)} \\
& \times\left(\lambda d \omega_{d}\left(\frac{r \sin \alpha}{\sin (\alpha+\beta)}\right)^{d}\left(\sum_{i=0}^{\lfloor(d-1) / 2\rfloor} b_{i}(\beta)\right)^{2}-\sum_{i=0}^{\lfloor(d-3) / 2\rfloor} c_{i}(\beta)\right) \\
& \times \exp \left\{-\lambda v_{d}(r, \alpha, \beta)\right\} \mathrm{d} \beta \mathrm{d} \alpha,
\end{aligned}
$$

and its value for $r=0$ is

$$
\begin{aligned}
f_{l_{d}}(0)= & \frac{d(d-1) \sqrt{\pi} \Gamma((d+1) / 2) \Gamma(d-1 / 2) \Gamma(2-2 / d) \lambda^{1 / d}}{2 \Gamma(d) \Gamma(2-1 / d)\{\Gamma((d+2) / 2)\}^{1+1 / d}} \\
& \times\left(2(d-1) \int_{0}^{\pi} \sin ^{d-2} \alpha\left(\sum_{i=0}^{\lfloor(d-1) / 2\rfloor} b_{i}(\alpha)\right)^{2} \mathrm{~d} \alpha-\sqrt{\pi} \frac{\Gamma((d+1) / 2)}{\Gamma((d+2) / 2)}\right) .
\end{aligned}
$$

See Figure 2. The moments corresponding to $F_{l_{d}}$ are

$$
\begin{gathered}
\mathrm{E} L_{d}^{k}=\frac{d(d-1) \Gamma(d-1 / 2) \Gamma((d+1) / 2) \Gamma(2+(k-1) / d) \pi^{d-1 / 2}}{2 \Gamma(d) \Gamma(2-1 / d)\{\Gamma((d+2) / 2)\}^{3-1 / d} \lambda^{k / d}} \\
\times \int_{0}^{\pi} \int_{0}^{\pi-\alpha} \frac{\sin ^{2 d-3} \alpha \sin ^{d-1} \beta}{\sin ^{2 d-1}(\alpha+\beta)} \\
\quad \times\left(\frac{(2 d+k-1) \pi^{d / 2} \sin ^{d} \alpha}{\Gamma((d+2) / 2) \sin ^{d}(\alpha+\beta)\left(v_{d}(1, \alpha, \beta)\right)^{3+(k-1) / d}}\left(\sum_{i=0}^{\lfloor(d-1) / 2\rfloor} b_{i}(\beta)\right)^{2}\right. \\
\left.\quad-\frac{\sum_{i=0}^{\lfloor(d-3) / 2\rfloor} c_{i}(\beta)}{\left(v_{d}(1, \alpha, \beta)\right)^{2+(k-1) / d}}\right) \mathrm{d} \beta \mathrm{d} \alpha .
\end{gathered}
$$

See Table 2. 


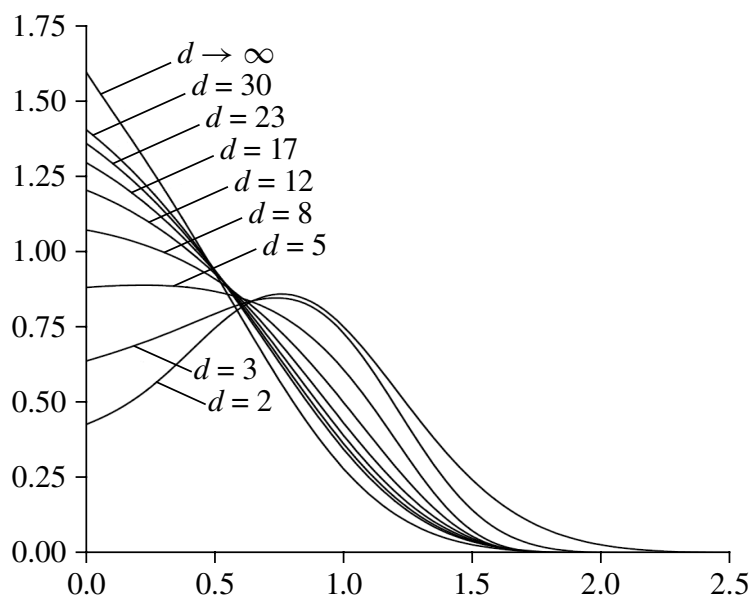

FIGURE 2: The probability density function of the chord length distribution of the Poisson-Voronoi tessellation in $\mathbb{R}^{d}, d=2,3,5,8,12,17,23,30, \infty$.

TABLE 2: Moments $\mathrm{E} L_{d}^{k}$ of the chord length distribution function of the Poisson-Voronoi tessellation in $\mathbb{R}^{d}, d=2,3,5,8,12,17,23,30, \infty$.

\begin{tabular}{clllll}
\hline \multirow{5}{*}{$d$} & \multicolumn{5}{c}{$k$} \\
\cline { 2 - 6 } & \multicolumn{1}{c}{1} & \multicolumn{1}{c}{2} & \multicolumn{1}{c}{3} & \multicolumn{1}{c}{4} & \multicolumn{1}{c}{5} \\
\hline 2 & 0.785398 & 0.806079 & 0.960132 & 1.273348 & 1.839899 \\
3 & 0.687182 & 0.631044 & 0.667220 & 0.773694 & 0.961232 \\
5 & 0.599056 & 0.501653 & 0.490882 & 0.528752 & 0.609437 \\
8 & 0.544325 & 0.429844 & 0.404484 & 0.423948 & 0.479079 \\
12 & 0.511349 & 0.388810 & 0.357746 & 0.370655 & 0.417491 \\
17 & 0.490587 & 0.363626 & 0.329690 & 0.339400 & 0.382409 \\
23 & 0.476813 & 0.347131 & 0.311468 & 0.319224 & 0.359914 \\
30 & 0.467239 & 0.335742 & 0.298915 & 0.305312 & 0.344355 \\
$\rightarrow \infty$ & 0.42888 & 0.29006 & 0.24783 & 0.24704 & 0.27612 \\
\hline
\end{tabular}

The last three formulae extend the results given in Muche and Stoyan [8], where formulae for $f_{l_{d}}(r), d=2,3$, and numerical results for $f_{l_{d}}(r), 2 \leq d \leq 7$, were given. They are alternative representations of the known general results given by Schlather [10, Equation (1.1)], and Muche [7, Equations (17)-(20)], where formulae for the length of the typical edge in an $n$-dimensional section for arbitrary $d$ were given ( $n=1$ represents chord lengths). Alishahi and Sharifitabar [1] presented an analytical expression for the limiting distribution function $F_{l_{\infty}}(r)$ and the value $f_{l_{\infty}}(0)$, whereas their curves for $f_{l_{d}}(r), 2 \leq d \leq 100$, were obtained by simulation only.

\section{Spherical contact distribution functions}

In this section we present a new approach to investigate the spherical contact distribution function $H_{s_{d, n}}$ for $2 \leq d<\infty$. All numbered formulae given here are new results in this 
generality. Earlier results were for the special cases $d=2$ and $d=3$ only; cf. [8]. Here the cases $n=1$ (linear section) and $n=d$ (complete tessellation $\mathcal{V}$ ) are investigated.

Case $n=1$. For $n=1$ (linear section), the test set $B$ is a centred line segment of length 2 . Consequently, $U_{r, \rho, \sigma}$ is the union of two overlapping balls with midpoint distance $2 r$ centred at the endpoints $-\underline{r}$ and $+\underline{r}$ of the straight line. Let the angles between $z_{0}$ and $r B$ at the endpoints of $r B$ be

$$
\beta_{1}=\angle\left(z_{0}, \underline{r}, o\right) \quad \text { and } \quad \beta_{2}=\angle\left(z_{0},-\underline{r}, o\right) .
$$

The probability density function of the spherical contact distribution $H_{s_{d, 1}}$ is given by

$$
\begin{aligned}
h_{s_{d, 1}}(r)= & \frac{2^{d+1} \pi^{d-1} \lambda^{2}}{\Gamma(d-1)} \\
& \times \int_{0}^{\pi / 2} \int_{\beta_{2}}^{\pi-\beta_{2}}(2 r)^{2 d-1} \frac{\left(\sin \beta_{1} \sin \beta_{2}\right)^{d-1}}{\sin ^{2 d}\left(\beta_{1}+\beta_{2}\right)} \\
& \times\left(\sin ^{d-1} \beta_{2} \sum_{i=0}^{\lfloor(d-1) / 2\rfloor} b_{i}\left(\beta_{1}\right)+\sin ^{d-1} \beta_{1} \sum_{i=0}^{\lfloor(d-1) / 2\rfloor} b_{i}\left(\beta_{2}\right)\right) \\
& \times \exp \left\{-\lambda v_{d}\left(2 r, \beta_{1}, \beta_{2}\right)\right\} \mathrm{d} \beta_{1} \mathrm{~d} \beta_{2} .
\end{aligned}
$$

There are close relationships between $H_{s_{d, 1}}(r)$ and $H_{l_{d}}(r)$ :

$$
H_{s_{d, 1}}(r)=H_{l_{d}}(2 r), \quad h_{s_{d, 1}}(r)=2 h_{l_{d}}(2 r), \quad \mathrm{E}_{s} R_{d, 1}^{k}=\frac{1}{2^{k}} \mathrm{E}_{l} R_{d}^{k} .
$$

Case $n=d$. For $n=d, B$ is the $d$-dimensional unit ball $B=b(o, 1)$ and $U_{r, \rho, \sigma}$ is the set of all balls centred at $b(o, r)$ with the point $z_{0}=(\rho, \sigma)$ on their boundaries. Let $v_{d}(r, \rho)$ denote the $d$-volume of $U_{r, \rho, \sigma}$. Its rotational symmetry with respect to the straight line passing through $z_{0}$ and $o$ ensures that $\nu_{d}(r, \rho)$ does not depend on $\sigma$. The set $U_{r, \rho, \sigma}$ can be described by the radius vector length

$$
l(r, \rho, \varphi)=\left\|z_{0}-u\right\|,
$$

where $u \in \partial U_{r, \rho, \sigma}$ and $\varphi=\angle\left(u z_{0} o\right)$ :

$$
l(r, \rho, \varphi)=\left\{\begin{array}{ll}
2(r+\rho \cos \varphi), & 0 \leq \varphi \leq \varphi^{*}, \\
0, & \varphi^{*} \leq \varphi \leq \pi,
\end{array} \quad 0 \leq r, \rho<\infty,\right.
$$

with

$$
\varphi^{*}= \begin{cases}\pi, & \rho \leq r \\ \pi-\arccos (r / \rho), & \rho>r .\end{cases}
$$

Note that in the special case $d=2$ the curve describing the boundary $\partial U_{r, \rho, \sigma}$ is concordant to the well-known limacon (snail) of Pascal. If $\rho \leq r$, it is the complete curve, otherwise it is the outer part; see Figure 3. In the case $d>2$ the corresponding object is the $(d-1)$-dimensional surface obtained by rotation of $U_{r, \rho, \sigma}$ for $d=2$ around its symmetry axis (line passing through $z_{0}$ and $o$ ).

Finally, $v_{d}(r, \rho)$ is given by

$$
v_{d}(r, \rho)=\kappa_{d} \sum_{i=0}^{d}\left(\begin{array}{l}
d \\
i
\end{array}\right) r^{d-i} \rho^{i} I_{i, d-2}\left(\varphi^{*}\right),
$$



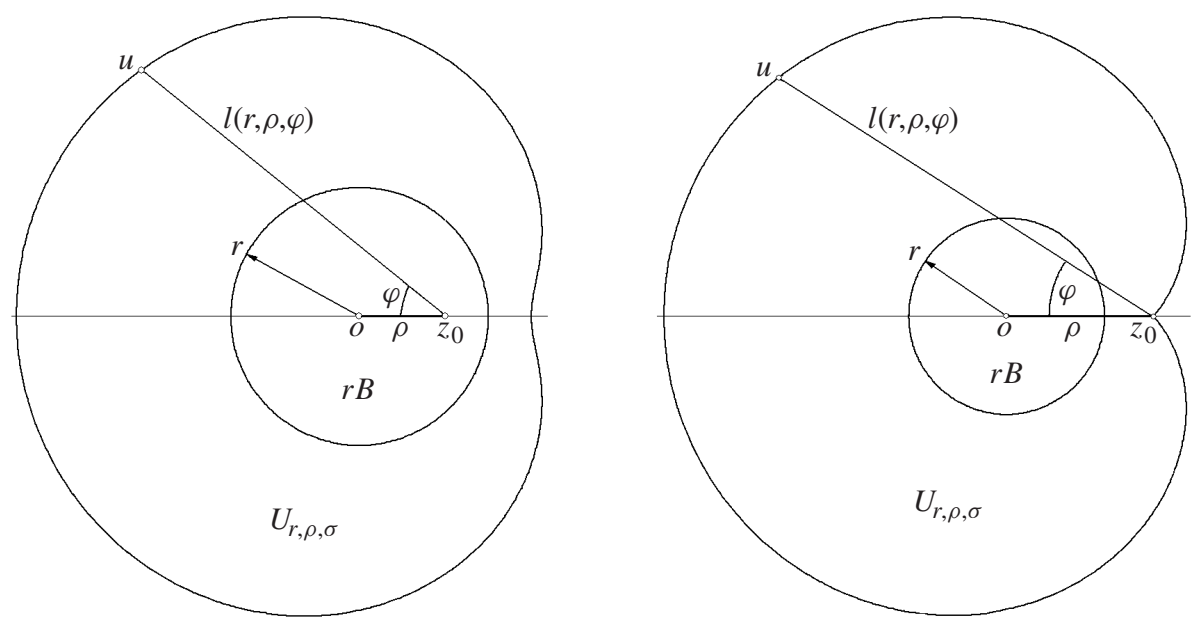

FIGURE 3: The curve describing $\partial U_{r, \rho, \sigma}$ in the planar case for $\rho<r$ (left) and $\rho>r$ (right).

where

$$
\kappa_{d}=\frac{2^{d+1} \pi^{(d-1) / 2}}{d \Gamma((d-1) / 2)},
$$

and

$$
I_{k, m}(\psi)=\int_{0}^{\psi} \cos ^{k} t \sin ^{m} t \mathrm{~d} t, \quad k, m \geq 0 .
$$

Its derivative with respect to $r$ is

$$
v_{d}^{\prime}(r, \rho)=d \kappa_{d} \sum_{i=0}^{d-1}\left(\begin{array}{c}
d-1 \\
i
\end{array}\right) r^{d-i-1} \rho^{i} I_{i, d-2}\left(\varphi^{*}\right) .
$$

The probability density function of the spherical contact distribution is given by

$$
h_{s_{d, d}}(r)=d \omega_{d} \lambda^{2} \int_{0}^{\infty} \rho^{d-1} v_{d}^{\prime}(r, \rho) \exp \left\{-\lambda v_{d}(r, \rho)\right\} \mathrm{d} \rho .
$$

Its value for $r=0$ is

$$
h_{s_{d, d}}(0)=\frac{2^{d+1} \Gamma(2-1 / d)\{\Gamma((d+2) / 2)\}^{2-1 / d} \lambda^{1 / d}}{d \Gamma(d-1 / 2)},
$$

with

$$
\lim _{d \rightarrow \infty} \frac{h_{s_{d, d}}(0)}{\sqrt{d}}=2 \sqrt{\mathrm{e} \pi} .
$$

See Figure 4. The moments corresponding to $H_{s_{d, d}}$ are

$$
\mathrm{E}_{s} R_{d, d}^{k}=\frac{\omega_{d} \Gamma(2+k / d)}{\lambda^{k / d}} \int_{0}^{\infty} \frac{\tau^{d-1} v_{d}^{\prime}(1, \tau)}{\left(v_{d}(1, \tau)\right)^{2+k / d}} \mathrm{~d} \tau, \quad k=0,1,2, \ldots
$$

See Table 3. 


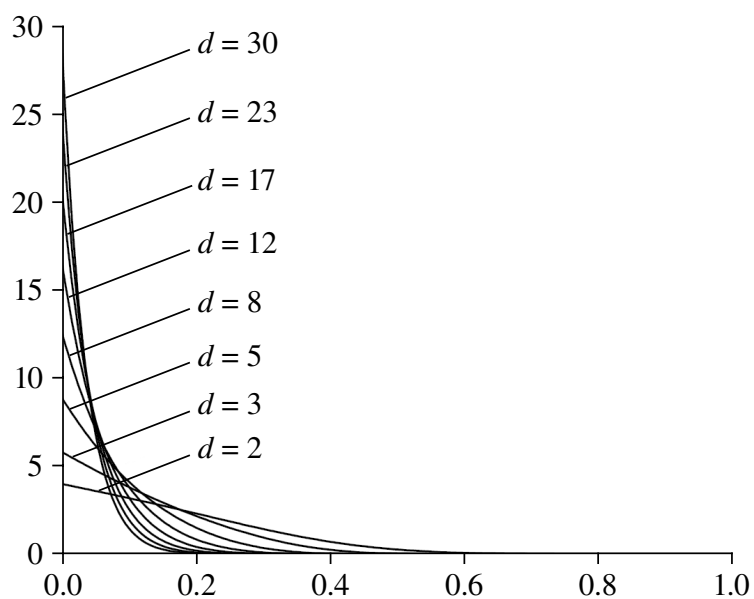

FIGURE 4: The probability density function corresponding to the spherical contact distribution function $H_{s_{d, d}}$ of the Poisson-Voronoi tessellation in $\mathbb{R}^{d}, d=2,3,5,8,12,17,23,30$.

TABLE 3: Moments $\mathrm{E}_{s} R_{d, d}^{k}$ of the spherical contact distribution function $H_{s_{d, d}}$ of the Poisson-Voronoi tessellation in $\mathbb{R}^{d}, d=5,8,12,17,23,30$.

\begin{tabular}{rccccc}
\hline \multirow{2}{*}{$d$} & \multicolumn{5}{c}{$k$} \\
\cline { 2 - 6 } & 1 & 2 & 3 & 4 & 5 \\
\hline 5 & 0.0915484 & 0.0141676 & 0.0028525 & $6.771 \times 10^{-4}$ & $1.803 \times 10^{-4}$ \\
8 & 0.0689896 & 0.0084292 & 0.0013861 & $2.756 \times 10^{-4}$ & $6.266 \times 10^{-5}$ \\
12 & 0.0546238 & 0.0054648 & $7.561 \times 10^{-4}$ & $1.294 \times 10^{-4}$ & $2.579 \times 10^{-5}$ \\
17 & 0.0449399 & 0.0037841 & $4.494 \times 10^{-4}$ & $6.715 \times 10^{-5}$ & $1.187 \times 10^{-5}$ \\
23 & 0.0380617 & 0.0027568 & $2.856 \times 10^{-4}$ & $3.769 \times 10^{-5}$ & $5.952 \times 10^{-6}$ \\
30 & 0.0329601 & 0.0020898 & $1.914 \times 10^{-4}$ & $2.254 \times 10^{-5}$ & $3.204 \times 10^{-6}$ \\
\hline
\end{tabular}

\section{Special cases}

In the following, the spherical contact distribution function $H_{s_{d, d}}(r)$ is investigated for the important cases $d=2$ and $d=3$. The distribution functions $H_{s_{2,2}}$ and $H_{s_{3,3}}$ have already been investigated in [8]. Here the probability density functions and moments for these two cases are given.

Planar case, $d=2$. The probability density function is given by

$$
\begin{aligned}
h_{s_{2,2}}(r)= & 4 \pi \lambda r\left(\exp \left\{-4 \pi \lambda r^{2}\right\}-\exp \left\{-6 \pi \lambda r^{2}\right\}\right) \\
+16 \pi \lambda^{2} \int_{r}^{\infty} & \rho\left(r\left(\pi-\arccos \frac{r}{\rho}\right)+\sqrt{\rho^{2}-r^{2}}\right) \\
& \times \exp \left\{-\lambda\left(\left(4 r^{2}+2 \rho^{2}\right)\left(\pi-\arccos \frac{r}{\rho}\right)+6 r \sqrt{\rho^{2}-r^{2}}\right)\right\} \mathrm{d} \rho .
\end{aligned}
$$


The corresponding moments are

$$
\begin{aligned}
\mathrm{E}_{s} R_{2,2}^{k}= & \Gamma\left(\frac{k}{2}+1\right)\left(\frac{1}{2(4 \pi \lambda)^{k / 2}}-\frac{1}{3(6 \pi \lambda)^{k / 2}}\right) \\
& +\frac{2 \pi \Gamma(k / 2+2)}{(2 \lambda)^{k / 2}} \int_{0}^{1} \frac{t^{k}\left(t(\pi-\arccos t)+\sqrt{1-t^{2}}\right)}{\left(\left(2 t^{2}+1\right)(\pi-\arccos t)+3 t \sqrt{1-t^{2}}\right)^{2+k / 2}} \mathrm{~d} t
\end{aligned}
$$

for $k=0,1,2, \ldots$ See Figure 5 and Table 4 .

Spatial case, $d=3$. The probability density function is given by

$$
\begin{aligned}
h_{s_{3,3}}(r)=\frac{64 \pi^{2} \lambda^{2} r^{5}}{3} \int_{0}^{1}( & 2 t^{2}\left(3+t^{2}\right) \exp \left\{-\lambda \frac{32 \pi}{3} r^{3}\left(1+t^{2}\right)\right\} \\
+ & \left.\frac{(1+t)^{3}}{t^{6}} \exp \left\{-\frac{4 \pi \lambda}{3} \frac{r^{3}}{t^{3}}(1+t)^{4}\right\}\right) \mathrm{d} t .
\end{aligned}
$$

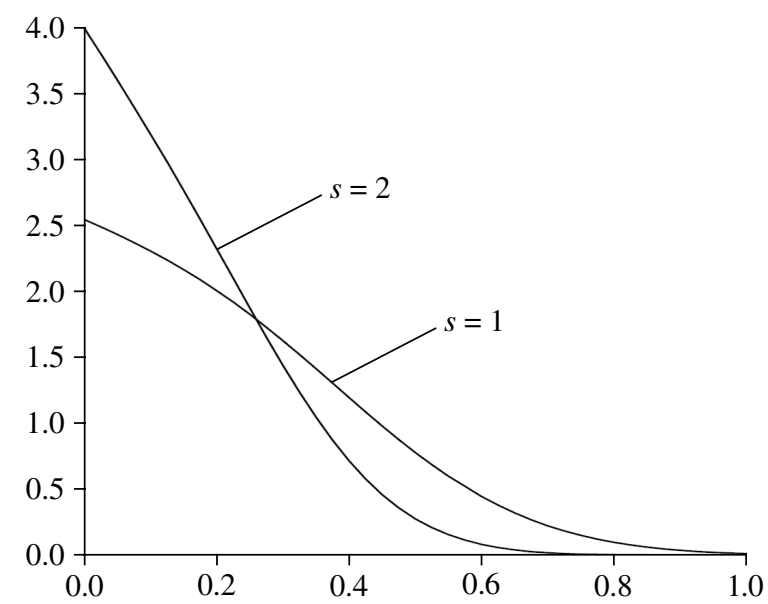

FIGURE 5: The probability density function corresponding to the spherical contact distribution function $H_{s_{d, s}}, s=1,2$, of the Poisson-Voronoi tessellation in $\mathbb{R}^{2}$.

TABLE 4: Moments $\mathrm{E}_{s} R_{d, s}^{k}, 1 \leq s \leq d$, of the spherical contact distribution function $H_{s_{d, s}}$ of the PoissonVoronoi tessellation in $\mathbb{R}^{2}$ and $\mathbb{R}^{3}$.

\begin{tabular}{ccccccc}
\hline \multirow{2}{*}{$d$} & \multirow{5}{c}{$k$} & \multicolumn{5}{c}{$k$} \\
\cline { 3 - 7 } & & 1 & 2 & 3 & 4 & 5 \\
\hline 2 & 1 & 0.256583 & 0.101873 & 0.050665 & 0.029283 & 0.018946 \\
2 & 2 & 0.174241 & 0.048046 & 0.016761 & 0.006829 & 0.003122 \\
3 & 1 & 0.229576 & 0.080912 & 0.035183 & 0.017485 & 0.009567 \\
3 & 2 & 0.158322 & 0.039811 & 0.012571 & 0.004583 & 0.001852 \\
3 & 3 & 0.127957 & 0.026363 & 0.006872 & 0.002078 & 0.000698 \\
\hline
\end{tabular}


The corresponding moments are

$$
\begin{aligned}
\mathrm{E}_{s} R_{3,3}^{k}= & \frac{1}{8}\left(\frac{3}{32 \pi \lambda}\right)^{k / 3} \Gamma\left(2+\frac{k}{3}\right) \\
\times & {\left[\frac{9}{2(k+3)} \mathcal{F}\left(\frac{k}{3}, \frac{1}{2}, \frac{3}{2},-1\right)-\frac{3}{2^{k / 3}(k+3)}+\frac{2^{k+5} k !}{\prod_{i=0}^{k}(k / 3+4+i)}\right.} \\
& \left.-\sum_{i=0}^{k} \frac{k !}{i !} \frac{2^{2 k / 3-i+1}}{\prod_{j=i}^{k}(k / 3+j+4)}\right], \quad k=0,1,2, \ldots,
\end{aligned}
$$

with the hypergeometrical function

$$
\mathcal{F}(\xi, \eta, \zeta, \tau)=\frac{\Gamma(\zeta)}{\Gamma(\eta) \Gamma(\zeta-\eta)} \int_{0}^{1} t^{\eta-1}(1-t)^{\zeta-\eta-1}(1-\tau t)^{-\xi} \mathrm{d} t,
$$

also known as the Gauss series or Kummer series,

$$
\begin{aligned}
\mathcal{F}(\xi, \eta, \zeta, \tau) & =\mathcal{F}(\eta, \xi, \zeta, \tau) \\
& =1+\sum_{i=1}^{\infty} \frac{\xi(\xi+1) \cdots(\xi+i-1) \eta(\eta+1) \cdots(\eta+i-1)}{i ! \zeta(\zeta+1) \cdots(\zeta+i-1)} \tau^{i}
\end{aligned}
$$

In the special case $k=3 m$ (for integer valued $m$ ) the moments can be given explicitly:

$$
\begin{aligned}
\mathrm{E}_{S} R_{3,3}^{3 m}= & \frac{1}{8}\left(\frac{3}{32 \pi \lambda}\right)^{m} \Gamma(m+2) \\
& \times\left[\frac{3}{8(m+1)}\left(\sum_{i=1}^{m-1} \frac{\prod_{j=i}^{m-2}((2 j+1) /(2 j+2))}{2^{i-1} i}+\pi \prod_{i=1}^{m-1} \frac{2 i-1}{2 i}\right)-\frac{1}{2^{m}(m+1)}\right. \\
& \left.+\frac{2^{3 m+5}(3 m) !}{\prod_{i=0}^{3 m}(m+i+4)}-\sum_{i=0}^{3 m} \frac{(3 m) !}{i !} \frac{2^{2 m-i+1}}{\prod_{j=i}^{3 m}(m+j+4)}\right], \quad m=1,2, \ldots ;
\end{aligned}
$$

in particular,

$$
\begin{aligned}
& \mathrm{E}_{s} R_{3,3}^{3}=\frac{9}{2048}+\frac{279}{35840 \pi}, \\
& \mathrm{E}_{s} R_{3,3}^{6}=\frac{3}{2464 \pi^{2}}+\frac{27}{65536 \pi}, \\
& \mathrm{E}_{s} R_{3,3}^{9}=\frac{81(139652+45045 \pi)}{41984983040 \pi^{3}}, \\
& \mathrm{E}_{s} R_{3,3}^{12}=\frac{81(591472+188955 \pi)}{563580239872 \pi^{4}} .
\end{aligned}
$$

See Figure 6 and Table 4. 


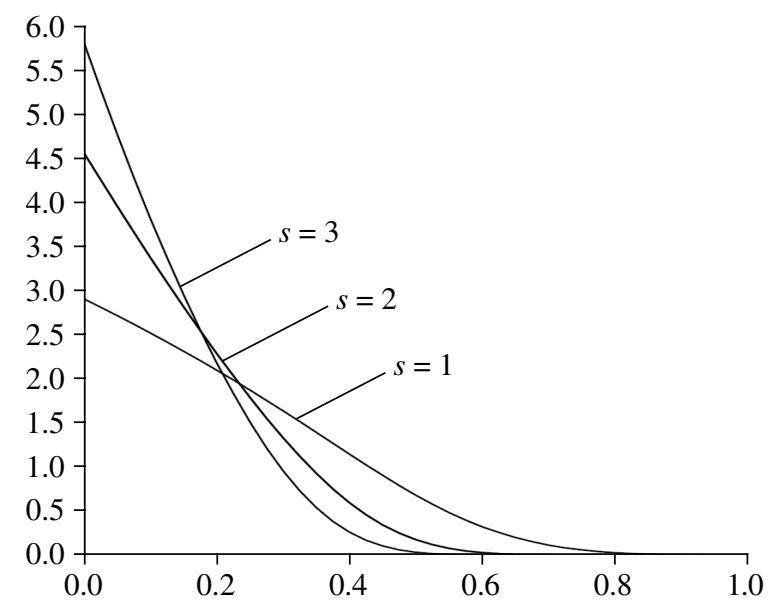

FIGURE 6: The probability density function corresponding to the spherical contact distribution function $H_{s_{d, s}}, 1 \leq s \leq 3$, of the Poisson-Voronoi tessellation in $\mathbb{R}^{3}$.

\section{Proofs}

Before starting the proofs, some integrals often used in the following are the generalized $\Gamma$-function,

$$
\int_{0}^{\infty} t^{p} \exp \left\{-q t^{\theta}\right\} \mathrm{d} t=\frac{1}{\theta} \Gamma\left(\frac{p+1}{\theta}\right)\left(\frac{1}{q}\right)^{(p+1) / \theta}, \quad p, q, \theta>0,
$$

and the trigonometric integral,

$$
\begin{aligned}
I_{k, m}(\psi)= & \int_{0}^{\psi} \cos ^{k} t \sin ^{m} t \mathrm{~d} t \quad \text { for integer valued } k, m \geq 0 \text { and real } \psi, 0 \leq \psi \leq \pi, \\
I_{k, m}(\psi)= & \frac{\sin ^{m+1} \psi}{2} \sum_{i=0}^{\lfloor(k-1) / 2\rfloor} \frac{\Gamma((k+1) / 2) \Gamma((k+m-2 i) / 2)}{\Gamma((k+m+2) / 2) \Gamma((k-2 i+1) / 2)} \cos ^{k-2 i-1} \psi \\
& + \begin{cases}\frac{\Gamma((k+1) / 2) \Gamma((m+2) / 2)}{\sqrt{\pi} \Gamma((k+m+2) / 2)} I_{0, m}(\psi), & k \text { even, } \\
0, & k \text { odd },\end{cases}
\end{aligned}
$$

with

$$
I_{0, m}(\psi)=\sqrt{\pi} \frac{\Gamma((m+1) / 2)}{\Gamma((m+2) / 2)} \sum_{i=0}^{\lfloor m / 2\rfloor} a_{i}(\pi-\psi), \quad m \geq 0,
$$

where $\lfloor\theta\rfloor$ denotes the greatest integer smaller or equal to $\theta$.

Formulae (36) and (37) can be obtained by integration by parts and recursion. In the particular cases of $\psi=\pi / 2$ and $\pi$, they simplify to

$$
\int_{0}^{\pi / 2} \cos ^{k} t \sin ^{m} t \mathrm{~d} t=\frac{1}{2} \frac{\Gamma((k+1) / 2) \Gamma((m+1) / 2)}{\Gamma((k+m+2) / 2)}
$$

and

$$
\int_{0}^{\pi} \sin ^{m} t \mathrm{~d} t=\sqrt{\pi} \frac{\Gamma((m+1) / 2)}{\Gamma((m+2) / 2)} .
$$


Furthermore, summation formulae for $\Gamma$-terms are

$$
\left\{\begin{array}{l}
\sum_{i=1}^{d / 2-1} \frac{\Gamma(i) \Gamma((m+2 i+1) / 2)}{\Gamma(i+3 / 2) \Gamma((m+2 i+2) / 2)} \\
\quad=\frac{8}{\sqrt{\pi} m} \frac{\Gamma((m+3) / 2)}{\Gamma((m+2) / 2)}-\frac{4}{m} \frac{\Gamma(d / 2)}{\Gamma((d+1) / 2)} \frac{\Gamma((d+m+1) / 2)}{\Gamma((d+m) / 2)}, \quad d \text { even, } d>2, \\
\sum_{i=1}^{d-1 / 2} \frac{\Gamma(i-1 / 2) \Gamma((m+2 i) / 2)}{\Gamma(i+1) \Gamma((m+2 i+1) / 2)} \\
=\frac{2 \sqrt{\pi} \Gamma(m / 2)}{\Gamma((m+1) / 2)}-\frac{4}{m} \frac{\Gamma(d / 2)}{\Gamma((d+1) / 2)} \frac{\Gamma((d+m+1) / 2)}{\Gamma((d+m) / 2)}, \quad d \text { odd, }
\end{array}\right.
$$

for $m>0$; see [7, Equation (30)], and Legendre's duplication theorem for $\Gamma$-terms is

$$
\Gamma(p) \Gamma\left(p+\frac{1}{2}\right)=\frac{\sqrt{\pi}}{2^{2 p-1}} \Gamma(2 p) .
$$

The relationship between Cartesian $\left(\xi_{1}, \xi_{2}, \ldots, \xi_{m}\right)$ and polar coordinates $\left(r, \varphi_{1}, \varphi_{2}, \ldots\right.$, $\left.\varphi_{m-1}\right)$ in $\mathbb{R}^{m}$ is

$$
\left\{\begin{array}{l}
\xi_{1}=r \cos \varphi_{1} \\
\xi_{i}=r \cos \varphi_{i} \prod_{j=1}^{i-1} \sin \varphi_{j}, \quad i=2, \ldots, m-1, \\
\xi_{m}=r \prod_{i=1}^{m-1} \sin \varphi_{i} \\
\prod_{i=1}^{m} \mathrm{~d} \xi_{i}=r^{m-1} \mathrm{~d} r \prod_{i=1}^{m-1}\left(\sin ^{m-i-1} \varphi_{i} \mathrm{~d} \varphi_{i}\right)
\end{array}\right.
$$

where $0 \leq \varphi_{i} \leq \pi, i=1, \ldots, m-2$, and $0 \leq \varphi_{m-1} \leq 2 \pi$.

Let $s(x, r w)$ be the line segment in $\mathbb{R}^{d}$ of length $r$ with endpoints $x$ and $x+r w$, and let $z_{0}=(\rho, \sigma)$ and $\sigma, w \in 8^{d-1}$. Then $s(x, r w)$ belongs to the origin cell $C_{0}$ if and only if the union $b\left(x,\left\|x-z_{0}\right\|\right) \cup b\left(x+r w,\left\|x+r w-z_{0}\right\|\right)$ does not contain any other point of $\Phi$. The convexity of $C_{0}$ implies that each further point $y=x+\mu r w, 0 \leq \mu \leq 1$, of the segment is also inside $C_{0}$. Therefore,

$$
\bigcup_{y \in s(x, r w)} b\left(y,\left\|y-z_{0}\right\|\right)=b\left(x,\left\|x-z_{0}\right\|\right) \cup b\left(x+r w,\left\|x+r w-z_{0}\right\|\right),
$$

and, consequently, for each compact convex set $Y \subset \mathbb{R}^{d}$,

$$
\bigcup_{y \in Y} b\left(y,\left\|y-z_{0}\right\|\right)=\bigcup_{y \in \partial Y} b\left(y,\left\|y-z_{0}\right\|\right) .
$$

See Figure 7. 


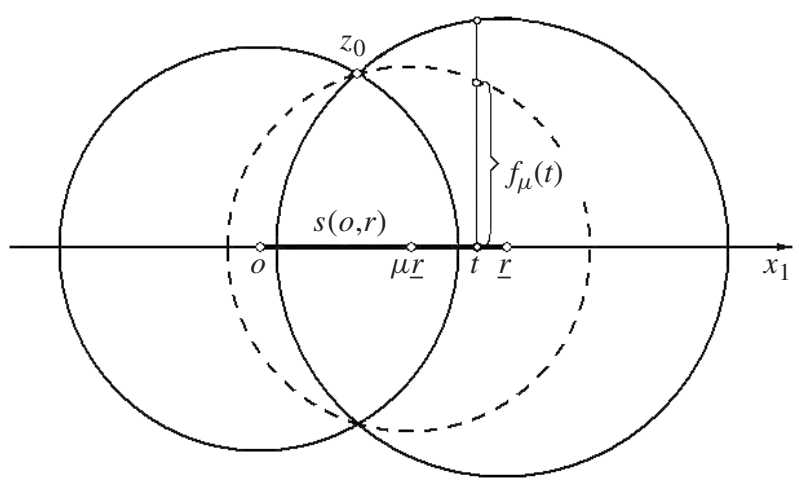

FIGURE 7: A line segment and two overlapping balls centred at its endpoints, the ball $b\left(\mu \underline{r},\left\|\mu \underline{r}-z_{0}\right\|\right)$ (dashed line) and its orthogonal distance $f_{\mu}(t)$ of the point with $x_{1}$-coordinate $t$; see (43). Here $o:=x$, $\underline{r}:=x+r w$, and $\mu \underline{r}:=y \in s[x, r w]$.

Proof of (13). Let $\sigma=\left(\sigma_{1}, \ldots, \sigma_{d}\right)$ be a point of $\varsigma^{d-1}$, and let $\mathscr{H}_{n}$ be the $n$-dimensional section subspace given by $x_{n+1}=\cdots=x_{d}=0$. Consequently, the projection $\sigma^{\prime}$ on $\mathscr{H}_{n}$ is $\sigma^{\prime}=\left(\sigma_{1}, \ldots, \sigma_{n}, 0, \ldots, 0\right)$. Denote that part of $\xi^{d-1}$ where $A_{d, n}=\angle \sigma o \sigma^{\prime}$ does not exceed a given value $\alpha, 0 \leq \alpha \leq \pi / 2$, by $\gamma_{\alpha}^{d-1}$. For all $\sigma \in \rho_{\alpha}^{d-1}$, the inequality $\sum_{i=n+1}^{d} \sigma_{i}^{2}<\sin ^{2} \alpha$ is true. Then the distribution function of the angle $A_{d, n}$ can be expressed as a ratio of $(d-1)$ volumes as follows:

$$
F_{A_{d, n}}(\alpha)=\frac{v_{d-1}\left(f_{\alpha}^{d-1}\right)}{v_{d-1}\left(8^{d-1}\right)} .
$$

For the sake of simplicity, consider only the first quadrant of $\varsigma^{d-1}$, where $\sigma_{1}, \sigma_{2}, \ldots, \sigma_{d} \geq 0$. Then $F_{A_{d, n}}(\alpha)$ can be written as

$$
\begin{aligned}
F_{A_{d, n}}(\alpha)= & \frac{2^{d}}{d \omega_{d}} \\
= & \int_{0 \leq \sigma_{n+1}, \ldots, \sigma_{d} ; \sum_{i=n+1}^{d} \sigma_{i}^{2} \leq \sin ^{2} \alpha}^{d-n} \int_{0 \leq \sigma_{i}<\sqrt{1-\sum_{j=i+1}^{d} \sigma_{j}^{2}}, i=2, \ldots, n}^{n-1} \\
& \times \prod_{i=2}^{d}\left(\mathrm{~d} \sigma_{i}\right) .
\end{aligned}
$$

To calculate the $(n-1)$-dimensional inner integral, the formula

$$
\int_{0}^{\theta}\left(\theta^{2}-t^{2}\right)^{m / 2} \mathrm{~d} t=\frac{\sqrt{\pi} \theta^{m+1}}{2} \frac{\Gamma((m+2) / 2)}{\Gamma((m+3) / 2)}
$$

is used, which follows from the substitution $t=\theta \sin \psi$ and the use of (39). We obtain

$$
\frac{\pi^{n / 2}}{2^{n-1} \Gamma(n / 2)}\left(1-\sum_{i=n+1}^{d} \sigma_{i}^{2}\right)^{n / 2-1} .
$$

The $(d-n)$-dimensional outer integral is solved by transformation to polar coordinates. If 
$n<d-1$, use of (42) for $m=d-n$ and $\sigma_{n+i}=\xi_{i}, i=1, \ldots, d-n$, gives

$$
F_{A_{d, n}}(\alpha)=\frac{2^{d-n} \Gamma(d / 2)}{\pi^{(d-n) / 2} \Gamma(n / 2)} \prod_{i=0}^{d-n-2}\left(\int_{0}^{\pi / 2} \sin ^{i} \varphi \mathrm{d} \varphi\right) \int_{0}^{\sin \alpha} r^{d-n-1}{\sqrt{1-r^{2}}}^{n-2} \mathrm{~d} r .
$$

Using (39) $d-n-1$ times allows us to determine the distribution function of the angle $A_{d, n}$ :

$$
F_{A_{d, n}}(\alpha)=\frac{2 \Gamma(d / 2)}{\Gamma(n / 2) \Gamma((d-n) / 2)} \int_{0}^{\sin \alpha} r^{d-n-1}{\sqrt{1-r^{2}}}^{n-2} \mathrm{~d} r .
$$

It can be shown that this result is also correct for $n=d-1$. Differentiation with respect to $\alpha$ yields (13).

Proofs of (14) and (15). The test set $B$ is the line segment of unit length starting at $o, B=$ $s(o, 1)$. Here $H_{B}(r)=H_{l_{d}}(r)$ is the probability that $r s(o, 1)=s(o, r)$ is not completely inside the origin cell $C_{0}$. Using (43), $U_{r, \rho, \sigma}$ is the union of the two balls centred at the endpoints of $s(o, r)$ with $z_{0}=(\rho, \sigma)$ on their boundaries. Using (3) and (7), replacing (4) by (13), and respecting the fact that $0 \leq \alpha \leq \pi$ gives

$$
H_{l_{d}}(r)=1-\frac{2 \pi^{(d-1) / 2} \lambda}{\Gamma((d-1) / 2)} \int_{0}^{\infty} \int_{0}^{\pi} \rho^{d-1} \sin ^{d-2} \alpha \exp \left\{-\lambda \nu_{d}(r, \alpha, \beta(r, \rho, \alpha))\right\} \mathrm{d} \alpha \mathrm{d} \rho,
$$

or by using (5) and the transformation formulae (9) and (10) we obtain

$$
H_{l_{d}}(r)=1-\frac{2 \pi^{(d-1) / 2} \lambda}{\Gamma((d-1) / 2)} \int_{0}^{\pi} \int_{0}^{\pi-\alpha} \frac{r^{d}(\sin \alpha \sin \beta)^{d-1}}{\sin ^{d+1}(\alpha+\beta)} \exp \left\{-\lambda \nu_{d}(r, \alpha, \beta)\right\} \mathrm{d} \beta \mathrm{d} \alpha .
$$

Differentiation of (45) with respect to $r$ leads to (14). Differentiation of (46) with respect to $r$ by use of (11), (12), and (41) gives (15).

Proofs of (16) and (17). Starting with (14), transform (5) and (11) by (8), and insert the limits $v_{d}(0, \alpha, \beta(0, \rho, \alpha))$ and $\lim _{r \downarrow 0}\left(\mathrm{~d} v_{d}(r, \alpha, \beta(r, \rho, \alpha)) / \mathrm{d} r\right)$. Use of (35), (39), and (40) for $m=d-1$ leads to (16).

To investigate $h_{l_{d}}(0)$ for large $d$, we use two well-known asymptotical properties of the $\Gamma$-function:

$$
\lim _{p \rightarrow \infty} \frac{\Gamma(p+1 / 2)}{\sqrt{p} \Gamma(p)}=1
$$

and Stirling's formula

$$
\Gamma(p+1)=\sqrt{2 \pi p}\left(\frac{p}{\mathrm{e}}\right)^{p} \mathrm{e}^{(\Theta / 12 p)}, \quad 0<\theta<1 .
$$

This gives (17).

Proof of (18). Use of (15), (35), and (41) gives (18).

Proof of (19). Two-fold differentiation of (1) with respect to $r$ gives

$$
f_{l}(r)=-\mathrm{E} L \frac{\mathrm{d}}{\mathrm{d} r}\left(h_{l}(r)\right)
$$


with the mean chord length

$$
\mathrm{E} L=\frac{d\{\Gamma((d+1) / 2)\}^{2} \Gamma(d-1 / 2)}{2 \Gamma(d) \Gamma(2-1 / d)\{\Gamma((d+2) / 2)\}^{2-1 / d} \lambda^{1 / d}} ;
$$

cf. also [2, Equation (9)]. Differentiation of (14) with respect to $r$ and insertion of (9) and (10) lead to (19).

Proofs of (20) and (21). We obtain (20) analogously to the proof of (16). Use of (35) gives (21).

Proof of (22). Analogously to the proof of (14), we obtain

$$
\begin{aligned}
H_{s_{d, 1}}(r)=1- & \frac{4 \pi^{(d-1) / 2} \lambda}{\Gamma((d-1) / 2)} \\
& \quad \times \int_{0}^{\infty} \int_{0}^{\pi / 2} \rho^{d-1} \sin ^{d-2} \alpha \\
& \quad \times \exp \left\{-\lambda v_{d}(b(-\underline{r},\|(\rho, \alpha)+\underline{r}\|) \cup b(+\underline{r},\|(\rho, \alpha)-\underline{r}\|))\right\} \mathrm{d} \alpha \mathrm{d} \rho .
\end{aligned}
$$

The $d$-volume in the exponent is equal to $v_{d}\left(2 r, \beta_{1}, \beta_{2}\right)$. Its derivative with respect to $r$ is calculated analogously to (11), respecting the fact that the set occurring in the exponent increases with $r$ in two directions, where $\pm \underline{r}$ denotes these points with coordinates $\pm r$ on the $x_{1}$-axis. Use of the transformation formulae

$$
\rho=\frac{r \sqrt{2\left(\sin ^{2} \beta_{1}+\sin ^{2} \beta_{2}\right)-\sin ^{2}\left(\beta_{1}+\beta_{2}\right)}}{\sin \left(\beta_{1}+\beta_{2}\right)}, \quad \alpha=\operatorname{arccot} \frac{\sin \left(\beta_{1}-\beta_{2}\right)}{2 \sin \beta_{1} \sin \beta_{2}},
$$

the Jacobian

$$
\left|\frac{\partial(\alpha, \rho)}{\partial\left(\beta_{1}, \beta_{2}\right)}\right|=4 r \frac{\sin \beta_{1} \sin \beta_{2}}{\sin ^{2}\left(\beta_{1}+\beta_{2}\right) \sqrt{2 \sin ^{2} \beta_{1}+2 \sin ^{2} \beta_{2}-\sin ^{2}\left(\beta_{1}+\beta_{2}\right)}},
$$

and (41) leads to (22). Application of symmetry arguments in (15) gives (23).

Proof of (24). Following (43) and (44), we have to determine

$$
U_{r, \rho, \sigma}=\bigcup_{w \in \AA^{d-1}} b\left(r w,\left\|r w-z_{0}\right\|\right)
$$

with $w=\left(w_{1}, w_{2}, \ldots, w_{d}\right)$ in Cartesian coordinates and the constraint $\sum_{i=1}^{d} w_{i}^{2}=1$. For the sake of simplicity, we assume that $z_{0}=(\rho, 0, \ldots, 0)$. We construct a function $l(r, \rho, \varphi)$ describing the distance between $z_{0}$ and the element $u$ of $\partial U_{r, \rho, \sigma}$ that satisfies $\varphi=\angle\left(u z_{0} o\right), 0 \leq$ $\varphi \leq \pi$. Rotational symmetry enables us to assume that $u$ is in the $\left(x_{1}, x_{2}\right)$-plane, i.e. $u=$ $(\rho-l \cos \varphi, l \sin \varphi, 0, \ldots, 0)$, where $l=l(r, \rho, \varphi)$. The value of $l(r, \rho, \varphi)$ is found via maximization of the distance $l_{w}=\left\|u_{w}-z_{0}\right\|$ for points $u_{w} \in \partial b\left(r w,\left\|r w-z_{0}\right\|\right)$. It is clearly $\left\|r w-u_{w}\right\|=\left\|r w-z_{0}\right\|$, i.e. the distance $\left\|u_{w}-z_{0}\right\|$ is $l(w)=2\left(\left(\rho-r w_{1}\right) \cos \varphi+r w_{2} \sin \varphi\right)$ for fixed $\varphi$. The wanted $u$ is the point $u_{w}$ that maximizes $l(w)$; see Figure 8. Replacing $w_{1}= \pm \sqrt{1-w_{2}^{2}}$ in $l(w)$, an elementary extreme value determination leads to (24), where the necessary inequality $l(r, \rho, \varphi) \geq 0$ leads to $\varphi^{*}$. 


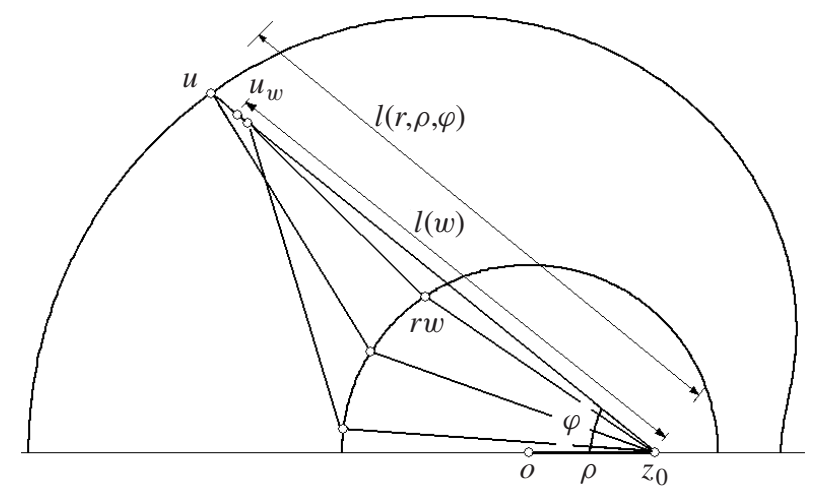

Figure 8: The point $u_{w}$ for various $w$ and fixed $\varphi$ with distance $l(w)=\left\|u_{w}-z_{0}\right\|$. Its maximum is the wanted function $l(r, \rho, \varphi)=\left\|u-z_{0}\right\|$.

Proofs of (25) and (26). Use of polar coordinates (42) gives the $d$-dimensional integral

$$
\nu_{d}(r, \rho)=\int^{d} l^{d-1} \mathrm{~d} l \prod_{i=1}^{d-1}\left(\sin ^{d-i-1} \varphi_{i} \mathrm{~d} \varphi_{i}\right)
$$

with $0 \leq l \leq 2\left(r+\rho \cos \varphi_{1}\right), 0 \leq \varphi_{1} \leq \varphi^{*}, 0 \leq \varphi_{i} \leq \pi, i=2,3, \ldots, d-2$, and $0 \leq \varphi_{d-1} \leq 2 \pi$. Repeated use of (39) leads to the $d$-volume of $U_{r, \rho, \sigma}$,

$$
v_{d}(r, \rho)=\kappa_{d} \int_{0}^{\varphi^{*}}(r+\rho \cos \varphi)^{d} \sin ^{d-2} \varphi \mathrm{d} \varphi,
$$

where $\varphi^{*}$ is given in (24), and its derivative with respect to $r$,

$$
v_{d}^{\prime}(r, \rho)=d \kappa_{d} \int_{0}^{\varphi^{*}}(r+\rho \cos \varphi)^{d-1} \sin ^{d-2} \varphi \mathrm{d} \varphi .
$$

Using (36) and (37), we obtain (25) and (26).

Proof of (27). Insert (3) and (25) into (2). Differentiation with respect to $r$ and insertion of (26) gives (27).

Proof of (28). Let $r=0$. Inserting (25) and (26) into (27), and using (38) and (35) leads to (28). Analogously to the proof of (17), by additional use of (41) we obtain (29).

Proof of (30). Substitution of $\rho=\tau r$ into (25), (26), and (27), and use of (35) leads to (30). For the special cases $d=2$ and $d=3$, we obtain

$$
\nu_{2}(r, \rho)=4 \begin{cases}\pi\left(r^{2}+\frac{\rho^{2}}{2}\right), & \rho<r, \\ \left(r^{2}+\frac{\rho^{2}}{2}\right)\left(\pi-\arccos \frac{r}{\rho}\right)+\frac{3}{2} r \sqrt{\rho^{2}-r^{2}}, & \rho \geq r\end{cases}
$$




$$
\begin{aligned}
& v_{2}^{\prime}(r, \rho)=4 \begin{cases}2 \pi r, & \rho<r, \\
2 r\left(\pi-\arccos \frac{r}{\rho}\right)+2 \sqrt{\rho^{2}-r^{2}}, & \rho \geq r,\end{cases} \\
& v_{3}(r, \rho)=\frac{16 \pi}{3} \begin{cases}2 r\left(\rho^{2}+r^{2}\right), & \rho<r, \\
\frac{(\rho+r)^{4}}{4 \rho}, & \rho \geq r,\end{cases} \\
& v_{3}^{\prime}(r, \rho)=\frac{16 \pi}{3} \begin{cases}2\left(3 r^{2}+\rho^{2}\right), & \rho<r, \\
\frac{(r+\rho)^{3}}{\rho}, & \rho \geq r,\end{cases}
\end{aligned}
$$

and, thus, we obtain the expressions for $h_{s_{2,2}}(r)$ and $h_{s_{3,3}}(r)$.

Proofs of (32) and (34). Inserting (47) and (48) into (27) and applying (35) gives

$$
\mathrm{E}_{S} R_{3,3}^{k}=\frac{1}{8}\left(\frac{3}{32 \pi \lambda}\right)^{k / 3} \Gamma\left(2+\frac{k}{3}\right)\left(\int_{0}^{1} \frac{t^{2}\left(3+t^{2}\right)}{\left(1+t^{2}\right)^{2+k / 3}} \mathrm{~d} t+2^{5+k} \int_{0}^{1} \frac{t^{k}}{(1+t)^{5+4 k / 3}} \mathrm{~d} t\right) .
$$

Integration by parts gives the recursion formulae

$$
\begin{aligned}
& \int_{0}^{1} \frac{t^{k}}{\left(1+t^{i}\right)^{m}} \mathrm{~d} t=\frac{-1}{2^{m+i-2}(m-1)}+\frac{k-i+1}{2^{i-1}(m-1)} \int_{0}^{1} \frac{t^{k-i}}{\left(1+t^{i}\right)^{m-1}} \mathrm{~d} t, \quad i=1,2, \\
& \int_{0}^{1} \frac{1}{\left(1+t^{2}\right)^{m}} \mathrm{~d} t=\frac{1}{2^{m}(m-1)}+\frac{2 m-3}{2 m-2} \int_{0}^{1} \frac{1}{\left(1+t^{2}\right)^{m-1}} \mathrm{~d} t, \\
& \int_{0}^{1} \frac{1}{(1+t)^{m}} \mathrm{~d} t=\frac{1}{(m-1)}\left(1-\frac{1}{2^{m-1}}\right),
\end{aligned}
$$

for $k, m>1$, which leads to

$$
\int_{0}^{1} \frac{t^{k}}{(1+t)^{5+4 k / 3}} \mathrm{~d} t=\frac{k !}{\prod_{i=0}^{k}(k / 3+4+i)}-\sum_{i=0}^{k} \frac{k !}{i !} \frac{1}{2^{k / 3+i+4} \prod_{j=i}^{k}(k / 3+j+4)} .
$$

Use of the substitution $t=t^{\prime 2}$ and (33) gives

$$
\begin{gathered}
\int_{0}^{1} \frac{t^{2}\left(3+t^{2}\right)}{\left(1+t^{2}\right)^{2+k / 3}} \mathrm{~d} t=-\frac{3}{2^{k / 3}(k+3)}+\frac{9}{2(k+3)} \mathcal{F}\left(\frac{k}{3}, \frac{1}{2}, \frac{3}{2},-1\right), \\
\mathcal{F}\left(m, \frac{1}{2}, \frac{3}{2},-1\right)=\sum_{i=1}^{m-1} \frac{\prod_{j=i}^{m-2}((2 j+1) /(2 j+2))}{i \cdot 2^{i+1}}+\frac{\pi}{4} \prod_{i=1}^{m-1} \frac{2 i-1}{2 i}, \quad m>0,
\end{gathered}
$$

and, thus, we obtain (32) and (34).

\section{Acknowledgements}

The author thanks Dietrich Stoyan for his encouragement and helpful discussions during the preparation of this paper. The author also thanks an anonymous referee for some valuable comments and suggestions. 


\section{References}

[1] Alishahi, K. and Sharifitabar, M. (2008). Volume degeneracy of the typical cell and the chord length distribution for Poisson-Voronoi tessellations in high dimensions. Adv. Appl. Prob. 40, 919-938.

[2] Gilbert, E. N. (1962). Random subdivisions of space into crystals. Ann. Math. Statist. 33, 958-972.

[3] Heinrich, L. (1998). Contact and chord length distribution of a stationary Voronoi tessellation. Adv. Appl. Prob. 30, 603-618.

[4] Last, G. AND Schassberger, R. (1998). On the distribution of the spherical contact vector of stationary germgrain models. Adv. Appl. Prob. 30, 36-52.

[5] Last, G. And Schassberger, R. (2001). On the second derivative of the spherical contact distribution function of smooth grain models. Prob. Theory Relat. Fields 121, 49-72.

[6] Muche, L. (1993). An incomplete Voronoi tessellation. Appl. Math. 22, 45-53.

[7] Muche, L. (2005). The Poisson-Voronoi tessellation: relationships for edges. Adv. Appl. Prob. 37, $279-296$.

[8] Muche, L. and Stoyan D. (1992). Contact and chord length distributions of the Poisson Voronoi tessellation. J. Appl. Prob. 29, 467-471. (Correction: 30 (1993), 749.)

[9] OKabe, A., Boots, B., Sugihara, K. And Chiu, S. N. (2000). Spatial Tessellations: Concepts and Applications of Voronoi Diagrams. John Wiley, Chichester.

[10] Schlather, M. (2000). A formula for the edge length distribution function of the Poisson Voronoi tessellation. Math. Nachr. 214, 113-119.

[11] Serra, J. P. (1982). Image Analysis and Mathematical Morphology. Academic Press, London.

[12] Stoyan, D., Kendall, W. S. And Mecke, J. (1995). Stochastic Geometry and Its Applications, 2nd edn. John Wiley, Chichester. 